University of South Carolina

Scholar Commons

$7-1-2004$

\title{
Synergetic Control of Power Converters for Pulse Current Charging of Advanced Batteries From a Fuel Cell Power Source
}

Zhenhua Jiang

University of Miami, zjiang1@miami.edu

Roger A. Dougal

University of South Carolina - Columbia, dougal@engr.sc.edu

Follow this and additional works at: https://scholarcommons.sc.edu/elct_facpub

Part of the Electrical and Computer Engineering Commons

Publication Info

Published in IEEE Transactions on Power Electronics, Volume 19, 2004, pages 1140-1150.

http://ieeexplore.iee.org/xpl/Recentlssue.jsp?punumber=63

(C) 2004 by IEEE

This Article is brought to you by the Electrical Engineering, Department of at Scholar Commons. It has been accepted for inclusion in Faculty Publications by an authorized administrator of Scholar Commons. For more information, please contact digres@mailbox.sc.edu. 


\title{
Synergetic Control of Power Converters for Pulse Current Charging of Advanced Batteries From a Fuel Cell Power Source
}

\author{
Zhenhua Jiang, Member, IEEE, and Roger A. Dougal, Senior Member, IEEE
}

\begin{abstract}
This paper presents a synergetic controller for pulse current charging of advanced batteries from a fuel cell power source. Pulse current charging protocol that has been shown to have many advantages over the traditional constant current/constant voltage protocol is applied in a fuel cell powered battery-charging station to reduce the total charging time. Strong nonlinearity and dynamics exist in such systems. In this paper, the synergetic control approach is applied to regulate the buck converters that control the pulse charging currents to the many batteries. A practical synergetic controller to coordinate pulse current charging of the battery is synthesized and discussed. It provides asymptotic stability with respect to the required operating modes, invariance to load variations, and robustness to variation of the input and converter parameters. The synergetic controller is then implemented in Simulink. The dynamic characteristics of the synergetic controller are studied and compared with PI controller by conducting system simulation and experimental tests. Simulation and experiment results show the synergetic controller is robust for such nonlinear dynamic system and achieves better performance than the standard PI controller.
\end{abstract}

Index Terms-Asymptotic stability, buck converters, PI controller, pulse current charging, synergetic controller.

\section{INTRODUCTION}

B ATTERY-POWERED portable electronic devices, such as portable computers, cellular phones and camcorders, are ubiquitous and now nearly essential in the daily lives of civilians and military personnel. Rechargeable batteries are playing an increasingly significant role in their utilization [1]. Their advantages, however, are partially restricted by the limited usable time, which requires a portable charging system in field applications. The fuel cell powered battery-charging station provides an attractive solution for these applications [2]-[4]. Power converters are used to condition the electrical power from the fuel cell stack to the batteries. The battery-charging station should generally allow multiple batteries to be charged simultaneously. It is therefore required that power converters be connected in parallel, each for one battery pack, with an additional advantage of enabling the flexible selection of the many charging protocols [2]-[4]. Recently, pulse current charging protocol has

\footnotetext{
Manuscript received June 10, 2003; revised December 19, 2003. This work was supported in part by the U.S. Army CECOM and the NRO under Contract NRO-00-C-0134, and by the U.S. ONR under Contract N00014-00-1-0368 and Contract N00014-00-1-0131. Recommended for publication by Associate Editor K. Ngo.

The authors are with the Department of Electrical Engineering, University of South Carolina, Columbia, SC 29208 USA.

Digital Object Identifier 10.1109/TPEL.2004.830044
}

been shown to have many advantages over the traditional constant current /constant voltage protocol. Pulse current charging, for example, can enhance the charging rate capability and prevent the increase of internal impedance of the battery, thus reducing the total charging time [5]. However, design of the controller for power converters in this application presents many challenges since their sources and loads are strongly nonlinear and the system is highly dynamic.

Many control methods have been applied to regulate power converters [6]-[10]. The classic proportional-integral control and linear negative feedback control are the most commonly used approaches. But these approaches require the linearization of the nonlinear controlled system. Other design methods, such as sliding mode variable structure control and one-cycle control, make use of the pulsed and nonlinear nature of switching converters and achieve instantaneous control of the average value of the chopped voltage or current. The objective of this paper is to apply a novel approach, synergetic control, in this particular system to overcome the problem described previously. Synergetic control theory was introduced in general terms in [11]. Its application to a single boost converter was introduced in [12], and some practical aspects with reference to both simulations and actual hardware were discussed in [13], [14].

In this paper, a synergetic controller for pulse current charging of advanced batteries from a fuel cell power source is developed and validated. The remainder of this paper is organized as follows. Section II discusses the control issues involved in this particular fuel cell/battery system. A practical and cost-effective synergetic control law to coordinate pulse current charging of the batteries is synthesized and discussed in Section III. Section IV presents the Simulink implementation of the synergetic controller for both system simulation and experimental tests. Simulation results and experiment validation are given in Section V. Conclusions are drawn in Section VI.

\section{Control Issues in the Fuel Cell/Battery CHARGING SYSTEM}

Fig. 1 conceptually shows the architecture of the proposed fuel cell powered battery- charging station that is suitable for many charging protocols (for instance, pulse current charging protocol or dc charging protocols) [2]-[4]. In the system, a fuel cell stack charges up to three lithium-ion battery packs through three separate buck converters, which represents the general case and provides the most flexibility in choosing the charging protocol. In other words, the user can easily change the charging protocol online with this multiconverter topology. Each battery contains four series-connected cells. Three buck converters are 


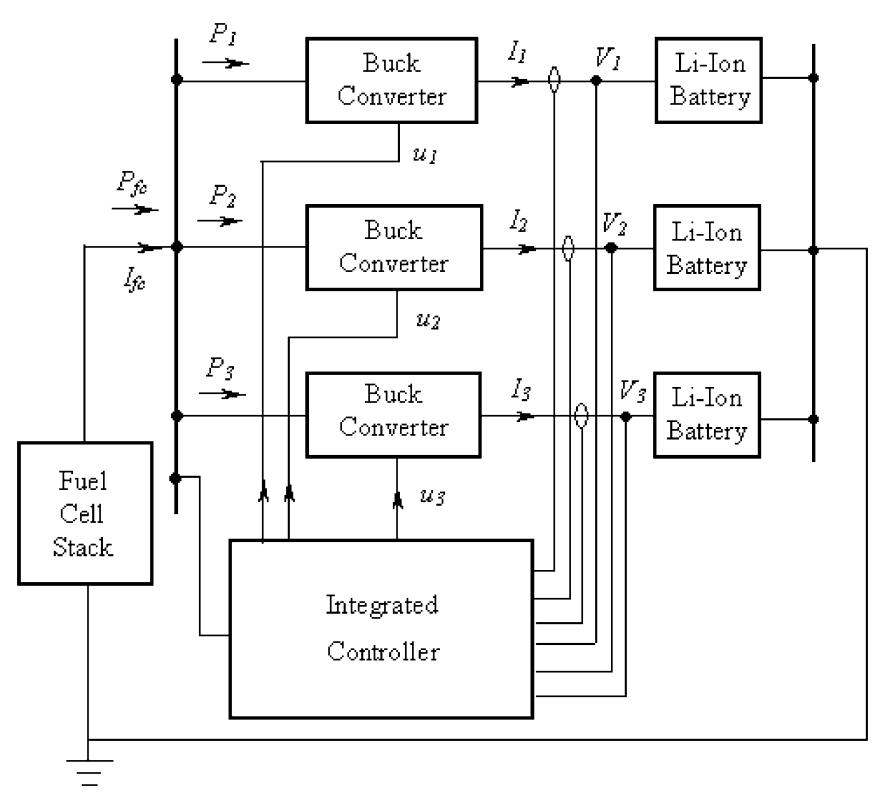

Fig. 1. Architecture of the proposed fuel cell/battery charging system.

connected in parallel to a single power source but they have different loads. The buck converters control the charging current or voltage of each battery, and allocate the available current from the power source among the batteries according to the pulse current charging strategy that is described in [2]. An integrated controller is used to regulate these buck converters.

With the pulse current charging strategy, three pulse currents with the same period of $T$ and different pulse-duration (note that the pulse-duration is different than the duty ratio of PWM switching signal) are applied to three batteries alternatively, as shown in Fig. 2. The sum of the pulse-durations of the three pulse currents is equal to the period $T$. The battery is charged at a higher rate $\left(I_{\text {high }}\right)$ during pulse ON while it is charged at a lower rate $\left(I_{\text {low }}\right)$ during pulse OFF. With such charging currents, three power converters are always active and the power available from the fuel cell power source can be fully utilized because each battery is able to share some amount of the current from the fuel cell at one time. A strategy is defined for the pulse current charging by making the pulse-duration of each pulse current proportional to the fraction of the depth-of-discharge of this battery. This pulse-duration can be estimated according to

$$
d_{i}=\frac{D O D_{i}}{D O D_{1}+D O D_{2}+D O D_{3}}, \quad i=1,2,3
$$

where $d_{i}$ is the pulse-duration of the charging current of the $i$ th battery, $D O D_{i}$ is the depth-of-discharge of the $i$ th battery that is defined as unity minus the state-of-charge of this battery.

An equivalent circuit for each of the three charging channels is illustrated in Fig. 3, where the parameter and state variable definitions are also given. When choosing the output capacitor voltage $v_{o}$ and inductor current $i_{L}$ as the state variables, the switching-averaged model of the buck converter is given by

$$
\begin{aligned}
& C \frac{d v_{o}}{d t}=i_{L}-i_{o} \\
& L \frac{d i_{L}}{d t}=u \cdot v_{S}-v_{o}
\end{aligned}
$$

where $u$ is the duty cycle of the PWM signal used to control the switching of the converter, $v_{s}$ is the fuel cell voltage, $L$ and $C$ are

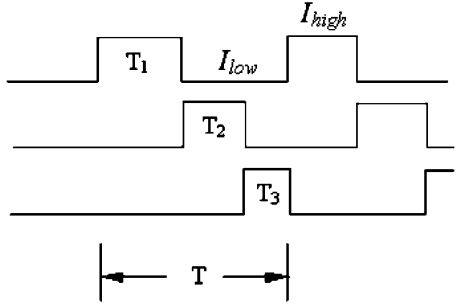

Fig. 2. Illustration of the pulse current charging strategy.

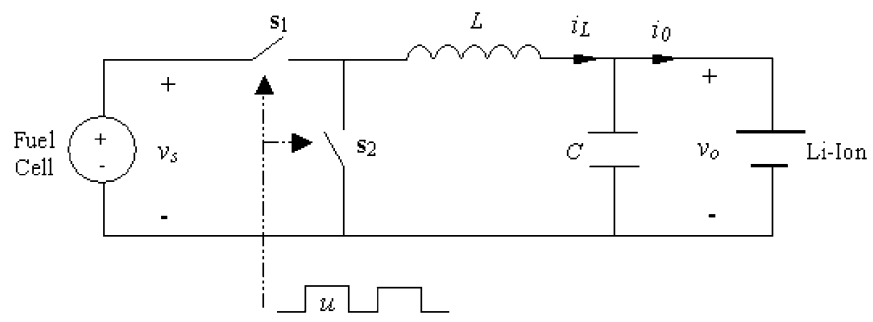

Fig. 3. Equivalent circuit of each charging channel of the fuel cell powered battery-charging station.

the inductance and capacitance of the output filter, and $i_{o}$ is the output current. Both the fuel cell and the battery are nonlinear electrochemical elements, and their electrical characteristics are simply expressed as

$$
\begin{aligned}
& v_{s}=f_{F C}\left(v_{s 0}, i_{F C}\right) \\
& v_{o}=f_{B}\left(i_{o}, S O C, t\right)
\end{aligned}
$$

where both $f_{F C}$ and $f_{B}$ are nonlinear functions, $v_{s 0}$ is the opencircuit voltage of the fuel cell stack, $i_{F C}$ and $i_{o}$ are, respectively, the currents from the fuel cell and to the battery, and $S O C$ is the battery state-of-charge that indicates the charge remaining in the battery.

As shown in Fig. 1, three such buck converters are connected in parallel to a single fuel cell power source. The active electrical power available from the fuel cell stack is distributed among these three batteries, which can be expressed in (6).

$$
P_{f c}=\frac{P_{1}}{\eta_{1}\left(P_{1}\right)}+\frac{P_{2}}{\eta_{2}\left(P_{2}\right)}+\frac{P_{3}}{\eta_{3}\left(P_{3}\right)}
$$

where $P_{1}, P_{2}$, and $P_{3}$ are the electrical power to three charging channels, respectively, and $P_{f c}$ is the electrical power available from the fuel cell stack, and $\eta_{2}, \eta_{2}$ and $\eta_{2}$ are, respectively, efficiencies of three buck converters and they are functions of the power delivered. In practice, the power distribution among the batteries is realized by regulating the charging currents of the batteries. The following equation relates the current available from the fuel cell stack to three charging currents approximately:

$$
I_{f c}=\frac{u_{1} I_{1}+u_{2} I_{2}+u_{3} I_{3}}{\eta_{b u c k}}
$$

where $I_{f c}$ is the current available from the fuel cell stack, $I_{1}, I_{2}$ and $I_{3}$ are, respectively, the currents of three charging channels, and $u_{1}, u_{2}$, and $u_{3}$ are, respectively, the duty cycles of three buck converters and they have values between 0 and $1, \eta_{B u c k}$ is the efficiency of the buck converter assuming that efficiency of each buck converter is identical and fixed.

From (1)-(7), it is seen that the fuel cell/battery charging system is strongly nonlinear and dynamic. In the following, 
the synergetic control approach is applied to coordinate these buck converters that control the pulse current charging of the batteries.

\section{SyNTHESIS OF THE SYNERGETIC CONTROL LAW}

The synergetic control design procedure follows the analytical design of aggregated regulators (ADAR) method. The general procedure for synergetic synthesis was described in [12]. A practical synergetic control law to regulate the pulse current charging of the battery is synthesized and discussed in this section

\section{A. Basic Synergetic Control Law}

Since buck converters are used to regulate the charging currents and voltages in the fuel cell powered battery-charging station, a basic synergetic control law for the buck converter is first studied. For convenience of demonstration at this point, in the equivalent circuit shown in Fig. 3, the source of the buck converter is replaced by a voltage source while the load by a resistor.

The synergetic synthesis of the controller begins by defining a macro-variable given in

$$
\Psi_{1}(t)=k_{1}\left(v_{o}-v_{r e f}\right)+k_{2}\left(i_{L}-i_{r e f}\right)
$$

where $k_{1}$ and $k_{2}$ are positive coefficients, $v_{r e f}$ and $i_{r e f}$ are the references of the output voltage and inductor current, respectively. The objective of the synergetic controller is to direct the system to operate on the manifold $\Psi_{1}(t)=0$.

Upon substitution of (8) into the following evolving equation:

$$
T_{1} \dot{\Psi}_{1}(t)+\Psi_{1}(t)=0, \quad T_{1}>0
$$

where $T_{1}$ is a controller parameter that indicates the converging speed of the closed-loop system to the manifold $\Psi_{1}(t)=0$, we can get

$$
k_{1} \dot{v}_{o}+k_{2} \dot{i}_{L}=-\frac{k_{1}\left(v_{o}-v_{r e f}\right)+k_{2}\left(i_{L}-i_{r e f}\right)}{T_{1}} .
$$

Directly substituting the governing (2) and (3) of the buck converter into (10) and rearranging, the control law is obtained which is given by

$u=\frac{v_{o}}{v_{S}}-\frac{k_{1} L\left(i_{L}-i_{o}\right)}{k_{2} v_{S} C}-\frac{k_{1} L\left(v_{o}-v_{r e f}\right)}{k_{2} T_{1} v_{S}}-\frac{L\left(i_{L}-i_{r e f}\right)}{T_{1} v_{S}}$.

Each manifold introduces a new constraint on the state space domain and reduces the order of the system by one, working in the direction of global stability. From the synergetic synthesis procedure described previously, it is clear that the synergetic controller works on the full nonlinear system and does not need any linearization or simplification on the system model at all as is necessary for application of traditional control theory. On the other hand, this is also a weakness: the synergetic control law theoretically requires precise knowledge of the model parameters. Such requirement places a serious limitation on the control system for two reasons: sometimes it is not easy to identify the parameters; sometimes the system parameters change with time. Here the situation where the load changes suddenly from one value of resistance to another is considered. This is particularly interesting because it is a typical problem for power electronics, where the power supply is supposed to compensate quickly for the load variation. As will be demonstrated later, this problem can be solved.

The performance of the basic synergetic control law under the condition of step load variation is tested through simulation. Both the buck converter, which is represented by (2) and (3), and the basic synergetic controller, which is described by (11), are modeled in Simulink, as shown in Fig. 4. The model parameters are listed in Table I. The control goal is to regulate $1 \mathrm{~A}$ average inductor current, and thus the reference of inductor current: $i_{r e f}=1.0 \mathrm{~A}$. The reference of the output voltage is specified based on the initial resistance of the load, which means that $v_{\text {ref }}=10.0 \mathrm{~V}$. These two reference values are not varied during the operation. The parameters of the controller are as follows: $k_{1}=0.001, k_{2}=1.0, T_{1}=0.00001$.

At $50 \mathrm{~ms}$, the load changes from nominal $10 \Omega$ to $8 \Omega$. The simulated inductor current and output voltage are shown in Fig. 5 (see the dashed lines). It is seen that the inductor current is disturbed by the step load variation, reaching a new steady-state value that is different from the desired $1 \mathrm{~A}$ reference. This is because, when a step load variation occurs, the resistance of the load changes suddenly whereas the model parameters in the control law do not change consequently. More specifically, the reference of the output voltage does not change with the load variation. Rather than exactly equal to their reference values, the final steady-state values of the inductor current and output voltage will be another pair of values that ensure (8) equals to zero when the system comes back to operate on the manifold $\Psi_{1}(t)=0$. It is also seen that there is a steady-state error in the output voltage following the step change in the load. This is a consequence of the fact that the inductor current offsets the reference value. If the change in the load increases, the steady-state error in these outputs will increase. It is desirable to reduce or eliminate the steady-state error in the outputs and thus the control law should be improved.

\section{B. Improved Synergetic Control Law}

In order to eliminate the steady-state error in the outputs, the following macro-variable is selected by introducing an integral error term of the inductor current in the macro-variable [13]

$$
\begin{aligned}
\Psi_{2}(t)=k_{1}\left(v_{o}-v_{r e f}\right)+k_{2}\left(i_{L}-\right. & \left.i_{r e f}\right) \\
& +k_{3} \int\left(i_{L}-i_{r e f}\right) d t
\end{aligned}
$$

where $k_{1}, k_{2}$ and $k_{3}$ are positive coefficients.

Upon substitution of (12) into the following evolving equation:

$$
T_{2} \dot{\Psi}_{2}(t)+\Psi_{2}(t)=0, \quad T_{2}>0
$$

where $T_{2}$ is a parameter of the controller that indicates the converging speed of the closed-loop system to the manifold $\Psi_{2}(t)=0$, and solving for $u$, we can get the following control law:

$$
\begin{aligned}
u & =\frac{v_{o}}{v_{S}}-\frac{k_{1} L\left(i_{L}-i_{o}\right)}{k_{2} v_{S} C}-\frac{k_{1} L\left(v_{o}-v_{r e f}\right)}{k_{2} T_{2} v_{S}} \\
& -\frac{\left(k_{2}+k_{3} T_{2}\right) L\left(i_{L}-i_{r e f}\right)}{k_{2} T_{2} v_{S}}-\frac{k_{3} L \cdot \int\left(i_{L}-i_{r e f}\right) d t}{k_{2} T_{2} v_{S}}
\end{aligned}
$$




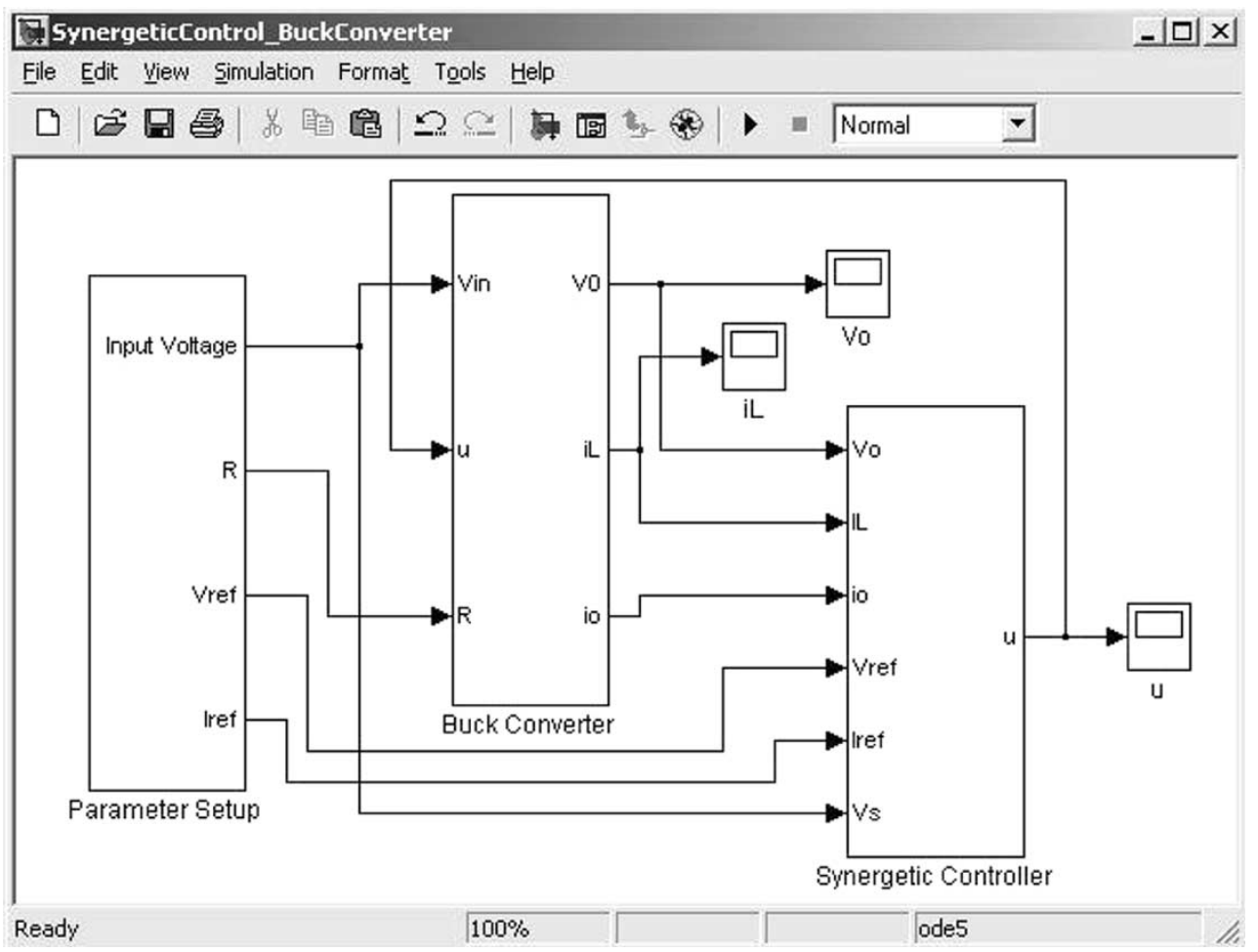

Fig. 4. Simulink model of a buck converter and a synergetic controller.

TABLE I

Model Parameters That ARE USEd IN THE SimUlation

\begin{tabular}{l|c|c|c}
\hline Nominal input voltage $V S$ & $20 \mathrm{~V}$ & Inductance $L$ & $470 \mathrm{uH}$ \\
\hline Nominal output current $I_{o}$ & $1 \mathrm{~A}$ & Capacitance $C$ & $10 \mathrm{uF}$ \\
\hline & & Initial Resistance $R$ & $10 \Omega$ \\
\hline
\end{tabular}
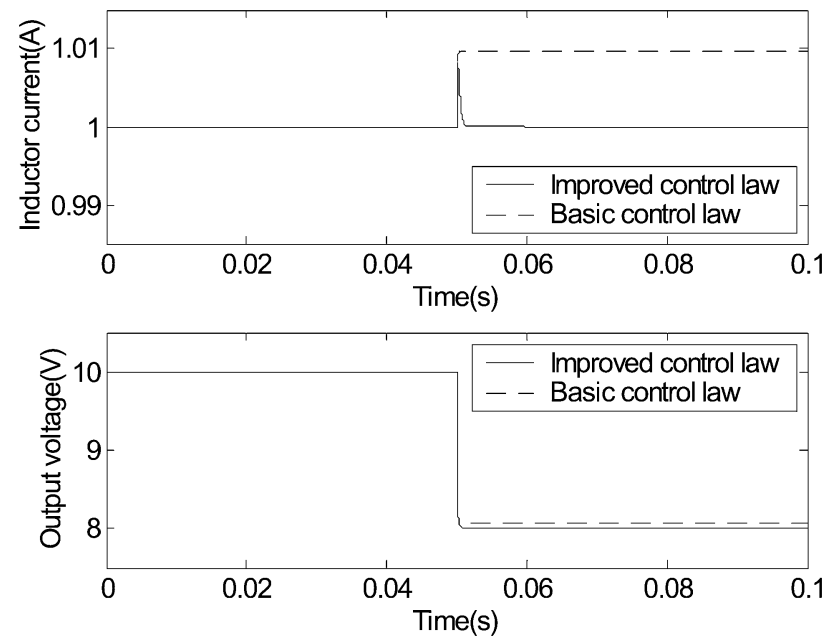

Fig. 5. Response to the step load change with the basic and improved synergetic control laws.

It is interesting to note that the control law in (14) consists of four distinct parts. The first part is the feedforward term, $v_{o} / v_{S}$, which is calculated based on the duty cycle at the previous sampling instant. This term compensates for variations in the input and output voltages. The second term consists of the difference between the slowly moving inductor current and the output cur- rent. If pole placement is at least one decade below the switching frequency, the inductor current ripple will be averaged by the control loop. Given the above pole placement, this term then approximates the perturbation in the output current. The third term contains proportional term involving the perturbations in the output voltage. The last two terms consist of proportional and integral terms of the perturbations in the inductor current respectively. From (14), it is also seen that the input voltage, output voltage, output current and inductor current are all involved in control output. The disadvantage is that the synergetic control approach requires more control inputs while the advantage is that it contributes better dynamic performance to the controlled system. Without the voltage feedback, the transient response would be very slow if the control goal were to stabilize the output current, since changes in the duty cycle would only take place with a perturbation in the output current away from the reference current.

The performance of the improved synergetic control law under the condition of step load change is also simulated. In the system shown in Fig. 4, the controller applies the control law given in (14). The parameters of the improved synergetic controller are as follows: $k_{1}=0.001, k_{2}=1.0, k_{3}=2500$, $T_{2}=0.00001$. The simulation results with the improved control law are also plotted in Fig. 5 for comparison (see the solid lines). It is shown that the steady-state error in the inductor 

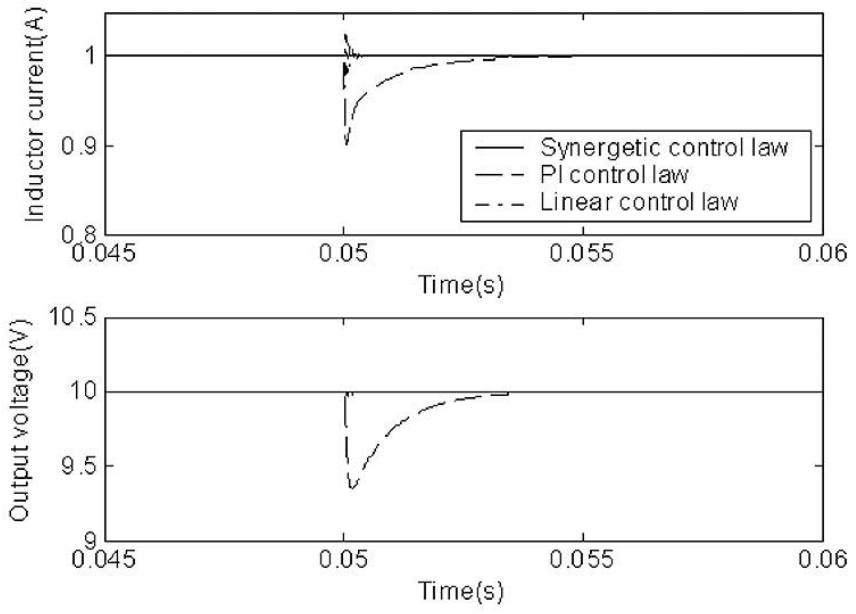

Fig. 6. Response to the step input voltage change with PI control law, higher order linear control law, and improved synergetic control law.

current is eliminated with the improved synergetic control law. The improved synergetic control law is more insensitive to the load variation compared with the basic control law. As a result of zero steady-state current error, there is also no steady-state error in the output voltage.

In the fuel cell powered battery-charging station, the input voltage will undergo frequent variations because the battery may be inserted or retrieved at any time, resulting in the rapid change in the output current of the fuel cell, and because there is a strong nonlinear relation between the current and voltage of the fuel cell. This is especially true when the pulse current charging protocol is applied to the batteries. The effect of the synergetic control law on suppressing the impact of the sudden change of the input voltage is then studied by comparing with two linear control approaches. Three control laws for identical buck converters are simulated: PI controller, higher-order linear compensator, and improved synergetic controller. The control law for the PI controller is

$$
u=k_{p}\left(I_{\text {ref }}-i_{L}\right)+k_{i} \int\left(I_{\text {ref }}-i_{L}\right) d t
$$

where $k_{p}$ is the proportional gain and $k_{i}$ is the integral gain. The parameters of the PI controller are as follows: $k_{p}=1.0$, $k_{i}=2500$. The transfer function of the higher-order linear compensator is $G_{c}(s)=1+\left(25000(s+0.1) / s^{2}-0.1 s+0.4\right)$. The parameters of the synergetic controller are as follows: $k_{1}=$ $0.001, k_{2}=1.0, k_{3}=2500, T_{2}=0.00001$.

The input voltage changes from nominal $22 \mathrm{~V}$ to $18 \mathrm{~V}$ at $50 \mathrm{~ms}$, but the load is constant. The simulated inductor currents and output voltages with the three control laws are plotted in Fig. 6 for comparison. It is seen that the inductor current and output voltage are disturbed by the step input voltage variation with the PI controller. The inductor current undergoes about $10 \%$ disturbance. Although the disturbance is not too significant here, it may become very large when the input voltage changes a lot. It takes $5 \mathrm{~ms}$ for the inductor current to reach the new steady state. The higher-order linear regulator reduces this disturbance to less than $2 \%$; however, there is ringing in the inductor current and output voltage, and the ringing lasts for about $1 \mathrm{~ms}$. With the synergetic controller, the inductor current does almost

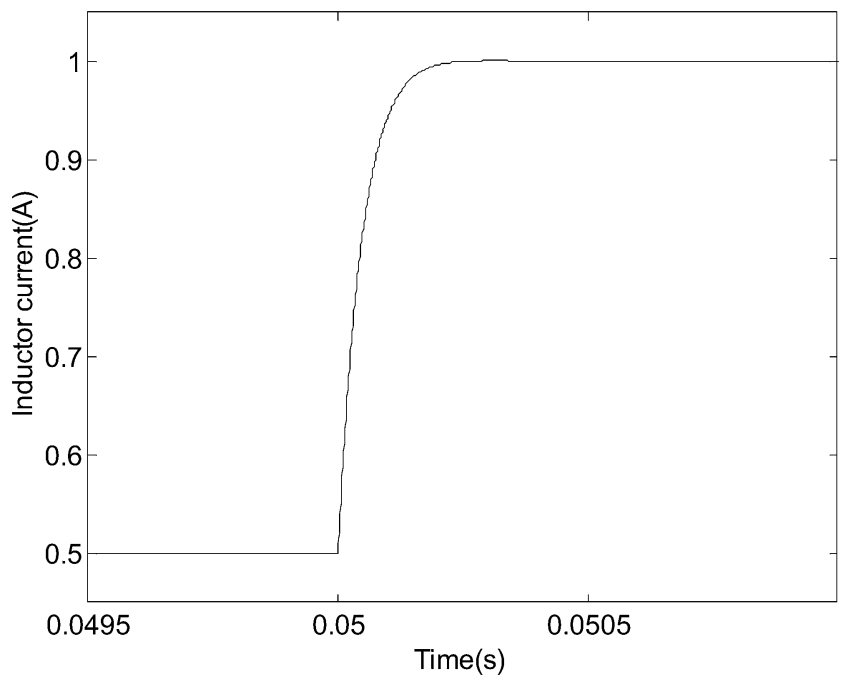

Fig. 7. Response to the step output current change with the improved synergetic control law.

not change any more. This is because the variation in the input voltage is immediately compensated by the first term of the improved synergetic control law that is given in (14). It is seen that the synergetic controller significantly suppresses any consequences of the sudden change of the input voltage.

When the pulse current charging protocol is applied in the fuel cell powered battery-charging station, a satisfactory response to the step output current change is desirable for the controller. The Simulink model shown in Fig. 4 is also used to study the response to step output current change with the synergetic controller. A small sinusoidal variation is added to the input voltage to represent a more practical situation. The reference output current changes from initial 0.5 A to $1.0 \mathrm{~A}$ at $50 \mathrm{~ms}$. The simulated inductor current, zoomed in between $49.5 \mathrm{~ms}$ and $51 \mathrm{~ms}$, is shown in Fig. 7. There is a little overshoot in the inductor current. This is because an integral term is introduced in the control law, increasing the order of the reduced-order system by one. The response time is about $0.25 \mathrm{~ms}$, which indicates that the response is very fast. It is seen that the synergetic controller is very robust to the step output current change.

\section{Synergetic Control for Pulse Current Charging}

From the simulation results shown above, it can be seen that the improved synergetic control law is less sensitive to the parameter variation and input variation. The improved synergetic control law is also robust to the step output current change. Due to these advantages, the synergetic control approach is used to regulate the pulse current charging of the batteries in the fuel cell powered battery-charging station.

In order to reduce the number of input variables of the control system, the control law in (14) is rearranged by combining with (2). The final control law is expressed as

$$
\begin{aligned}
u & =\frac{v_{o}}{v_{S}}-\frac{k_{1} L \dot{v}_{o}}{k_{2} v_{S}}-\frac{k_{1} L\left(v_{o}-v_{r e f}\right)}{k_{2} T_{2} v_{S}} \\
& -\frac{\left(k_{2}+k_{3} T_{2}\right) L\left(i_{L}-i_{r e f}\right)}{k_{2} T_{2} v_{S}}-\frac{k_{3} L \cdot \int\left(i_{L}-i_{r e f}\right) d t}{k_{2} T_{2} v_{S}}
\end{aligned}
$$




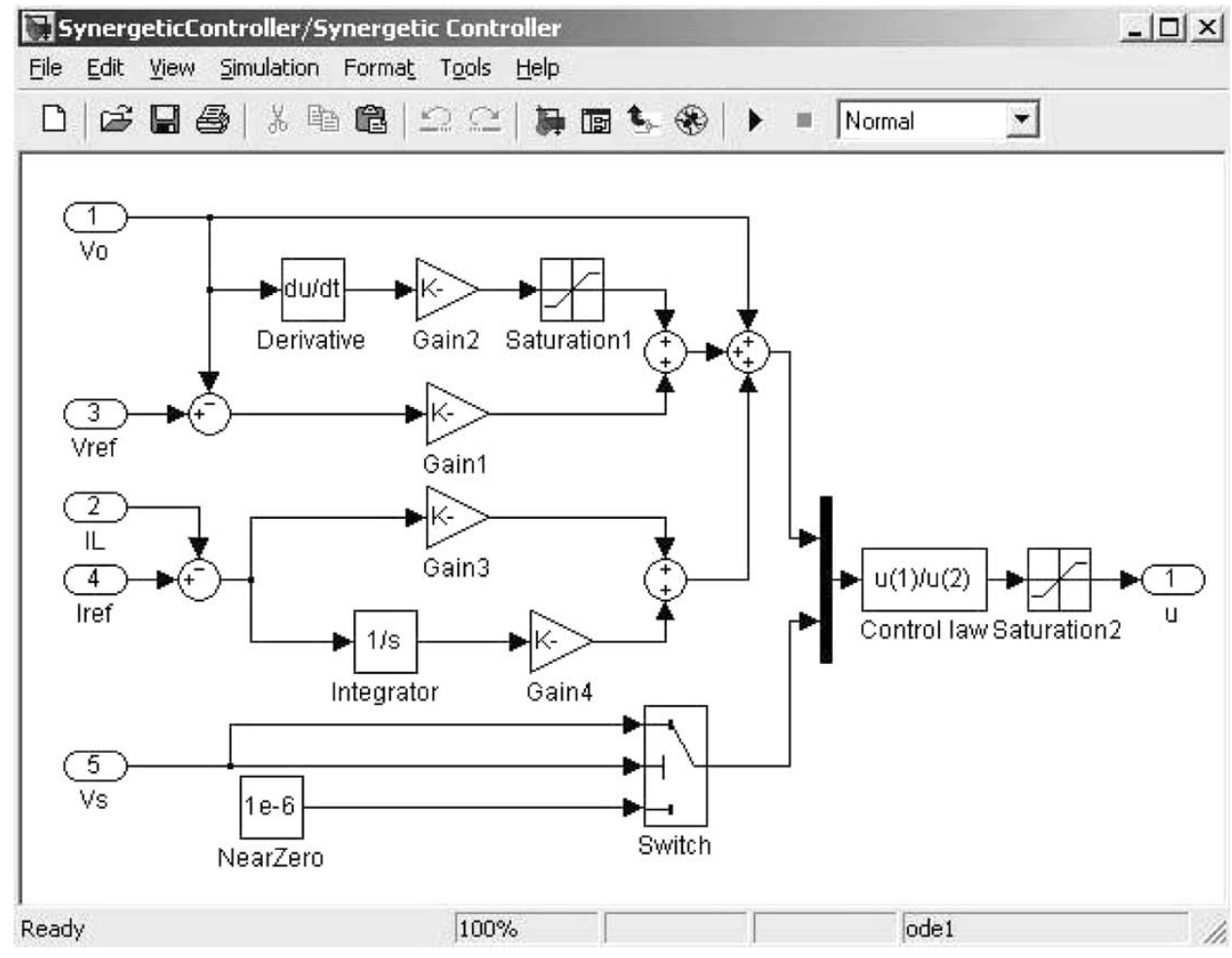

Fig. 8. Simulink model of the synergetic controller for the pulse current charging of each battery.

It is interesting to note that the control scheme in (16) that is calculated according to synergetic control theory is actually a combination of feed-forward control, multiloop control, and nonlinear proportional-integral (PI) and proportional-derivative (PD) controls. The control system has three input variables: input voltage, inductor current and output voltage; thus it is not difficult to implement the control scheme on the hardware. A Simulink diagram of the synergetic controller for the pulse current charging of each battery is shown in Fig. 8. The inputs of this controller are the three input variables shown above and the voltage and current reference values. The output is the duty cycle of the buck converter. This controller can be used later in the simulation studies and experiment tests by rearranging the input/output ports properly. Compared with the linear regulators, the synergetic controller needs only one more input variable (the voltage of the power source) but achieves better transient response, as will also be shown in the experiments.

\section{SIMULINK IMPLEMENTATION OF THE SYNERGETIC CONTROLLER}

The synergetic controller is implemented in MATLAB/Simulink that is convenient both for system simulation and for experimental tests. In this study, system simulations are conducted in virtual test bed (VTB) [15] simulation environment by embedding the Simulink object of the controller into VTB and cosimulating interactively with MATLAB. Experimental tests are performed by compiling the Simulink codes of the controller and downloading to the dSPACE platform to control the actual hardware.
The Simulink model of the synergetic controller for the fuel cell powered battery-charging station is shown in Fig. 9. There are seven input terminals and six output terminals in this Simulink model. The measured charging currents and voltages of three batteries and the voltage of the fuel cell stack are input from the VTB environment or the hardware through seven input terminals. The reference charging current for each battery and the corresponding duty cycle for each power converter are calculated in this Simulink model. The charging controller model also determines the turn-on or turn-off of the switch connected to each battery. The controller can output a turnoff signal to isolate the battery when the charging stops or when a fault is detected. The calculated values of the duty cycles and the switch states for three charging channels are exported to the VTB environment or to hardware through six output terminals.

The main functional blocks in this charging controller are the charging current strategy module, synergetic regulation module, and charging termination decision module. The charging current strategy module is responsible for calculation of the pulse-durations of the charging currents according to the measured voltages and currents of the batteries and the voltage of the fuel cell stack. It is developed based on the control strategy for active power sharing shown in (1). The estimation of the state-ofcharge is also implemented in this module. The outputs of this module are the reference charging currents for three batteries. The synergetic regulation module is used to compute the duty cycles of PWM signals to the buck converters according to the reference currents and reference voltages from the charging current strategy module, the measured voltages and currents of the batteries and the voltage of the fuel cell stack, as shown in Fig. 8. The charging termination decision module determines when the 


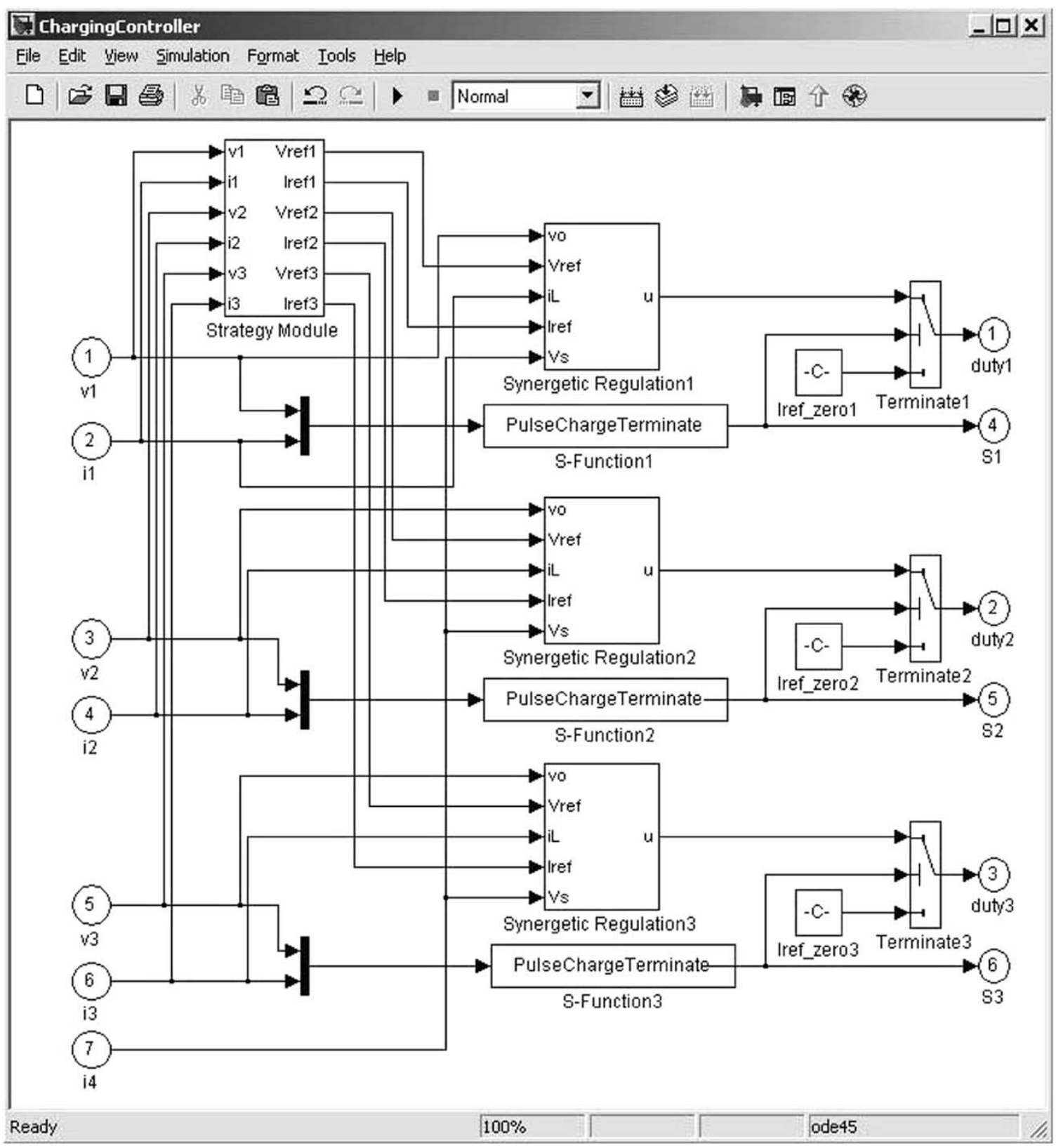

Fig. 9. Simulink implementation of the synergetic controller for the fuel cell powered pulse-current charging station for three batteries.

charging process should terminate and then outputs a switching signal to the corresponding charging channel.

\section{Simulation Study AND EXPERIMENT VALIDATION}

\section{A. Simulation Environment}

In order to investigate the performance of the synergetic controller, a virtual prototype is first assembled in the VTB that provides mechanisms for importing models from MATLAB and cosimulating with Simulink. Fig. 10 shows the virtual prototype of the fuel cell powered battery-charging station. This system consists of a fuel cell stack, three battery packs, three buck converters and a charging controller. The power source is a 25-cell PEM fuel cell stack. An input filter is connected between the fuel cell stack and the main power bus. Each battery is a $4 \times 1$ (series by parallel connections) array of lithium-ion cells. The capacity of each battery is $1.5 \mathrm{Ah}$. Each power converter is represented as a switching-average buck converter model in series with a low pass filter. The charging controller that is implemented in Simulink, as shown in Fig. 9, is compiled into a model that can run within the VTB environment.

\section{B. Experiment Configuration}

A hardware prototype of the fuel cell powered battery-charging station is also designed and built. The configuration of the experiment environment is shown in Fig. 11. The power source is an H Power D35 PEM fuel cell stack, which has a nominal power capacity of $35 \mathrm{~W}$ and an open-circuit voltage of $24 \mathrm{~V}$. It charges up to three lithium-ion battery packs through a power processing circuit that contains three individual buck converters. Each battery contains four series-connected Panasonic lithium-ion cells. Each cell has a 


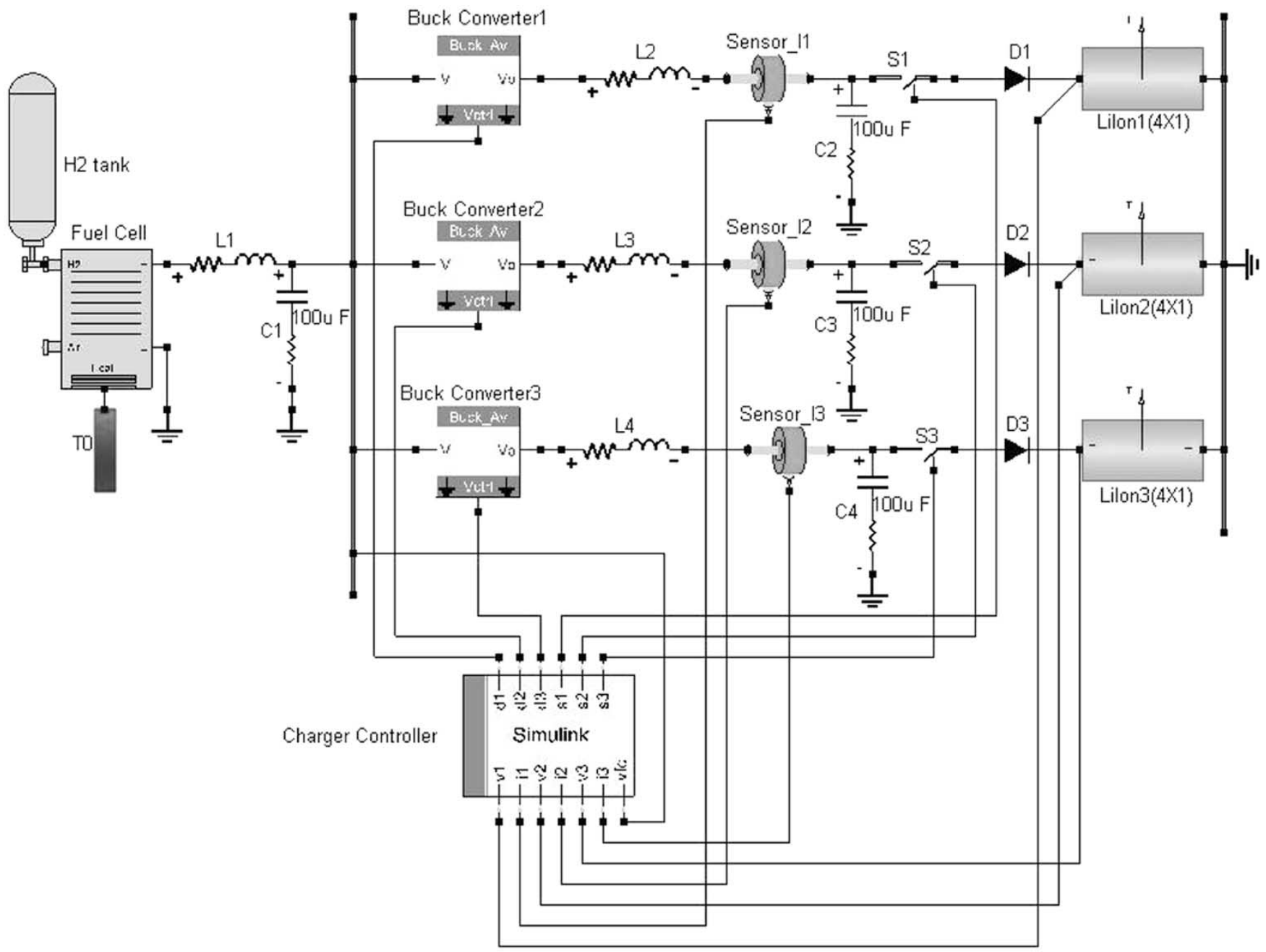

Fig. 10. Virtual prototype of the fuel cell powered battery-charging station in the VTB environment.

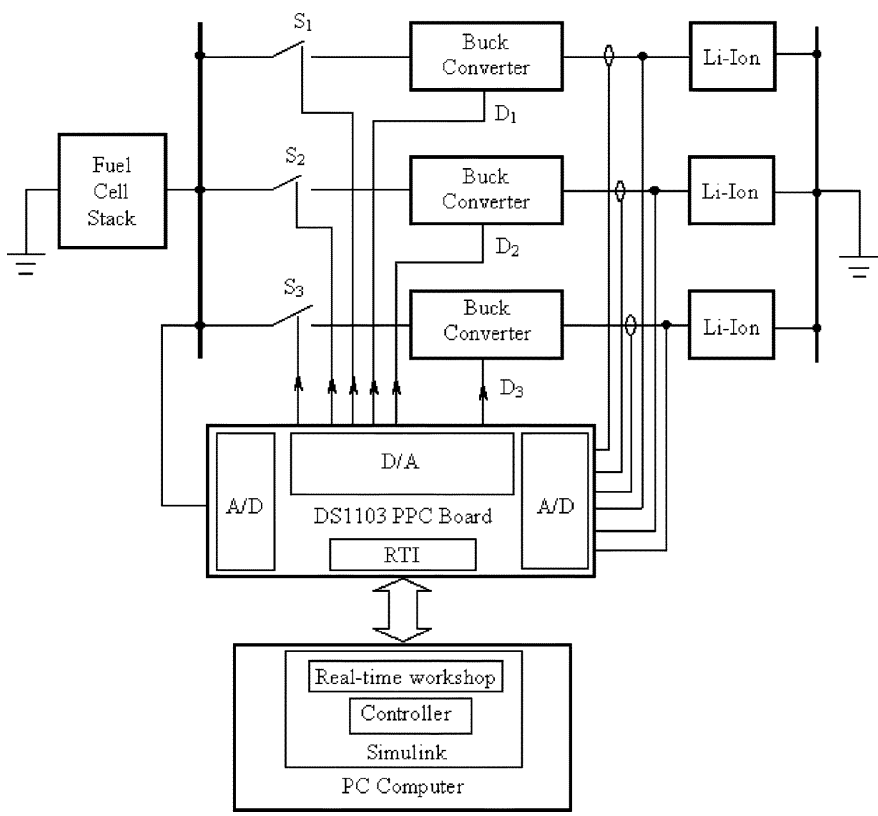

Fig. 11. Configuration of the experiment environment.

nominal voltage of $3.6 \mathrm{~V}$ and a capacity of $1.5 \mathrm{Ah}$. The three identical buck converters are connected in parallel to the same fuel cell stack. The switching frequency of each buck converter is $100 \mathrm{kHz}$. The charging control algorithm executes on a general-purpose dSPACE controller board, which also houses the hardware interface consisting of multichannel $\mathrm{A} / \mathrm{D}$ and $\mathrm{D} / \mathrm{A}$ converters. The control algorithm is compiled directly from the MATLAB/Simulink model and then downloaded to dSPACE controller board (model DS1103 PPC).

The charging currents and battery voltages are monitored and input to the DS1103 PPC controller board through the A/D converters mounted on it. The power source bus voltage is also an input variable. The real-time controller provides the switch duty commands to each power converter. The circuit protection functions-limiting the fuel cell current, battery current and battery voltage-are implemented in the software that is executed on the DS1103 PPC controller board. The specifications of the system are the same as those in simulations.

\section{Results and Discussions}

Tests are conducted on the experiment platform described previously in two scenarios: with a single battery and with three batteries. In both cases, the reference current for each battery is a pulse current. The high value of the pulse is $1.5 \mathrm{~A}$ while the low 


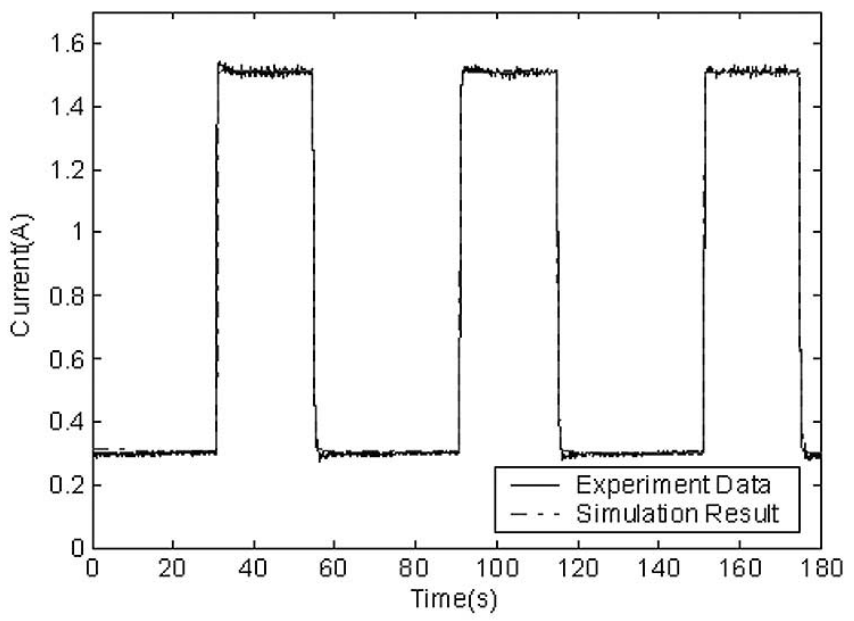

(a)

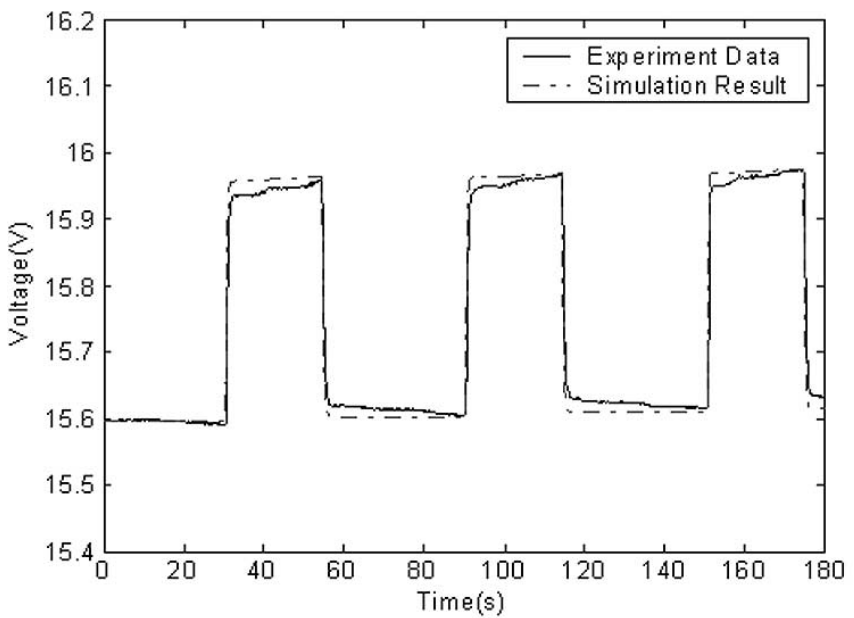

(b)

Fig. 12. Experimental data and simulation results of the charging current and voltage of a single battery with the synergetic controller: (a) current and (b) voltage.

value is $0.3 \mathrm{~A}$, which means that when one battery is charged at higher rate $(1.5 \mathrm{~A})$ the other two batteries are charged at a lower rate $(0.3 \mathrm{~A})$. The period of the pulse is $1 \mathrm{~m}(60 \mathrm{~s})$.

Fig. 12 shows experiment data and simulation results of the charging current and battery voltage as a single battery is charged. Here, the duty ratio of the reference pulse current is $40 \%$, i.e., the pulse duration is $24 \mathrm{~s}$. It is seen from Fig. 12(a) that the current response is quite close to the reference and the behavior of the controller is good. There is a small over-shoot following the rising or falling edge of the current. This is because an integral term is introduced in the control law, increasing the reduced system order (first order) by one. The battery voltage is also in a pulsing form, as shown in Fig. 12(b). This is due to the equivalent series resistance (ESR) of the battery and the pulse current. The battery voltage increases during the pulse-on time and decreases slightly during the pulse-off time. From the view of a long span of time, the battery voltage increases gradually. It can be seen that experiment data match the simulation results very well. The unclean waveforms result from the difference between switching frequency $(100 \mathrm{kHz})$ and sampling frequency $(20 \mathrm{kHz})$, although low-pass filters

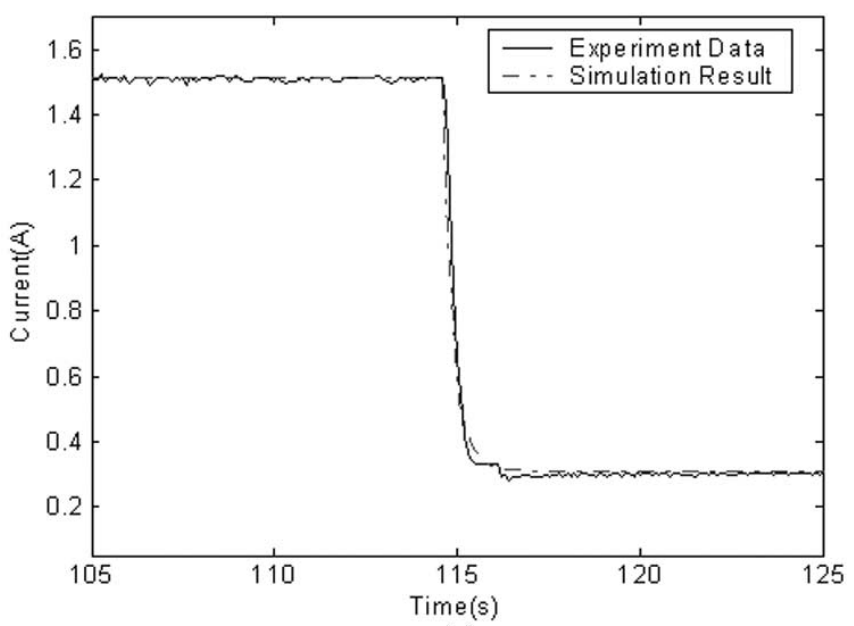

(a)

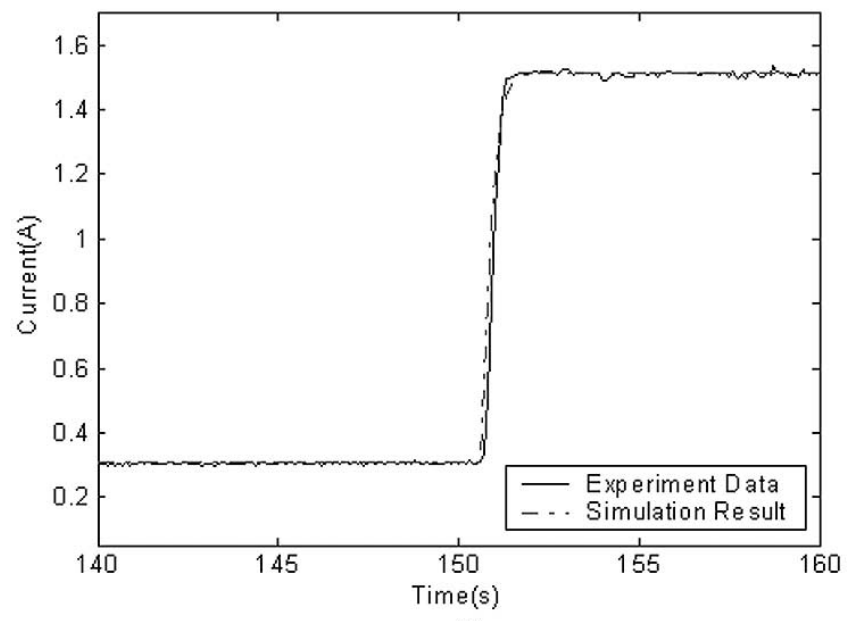

(b)

Fig. 13. Zoomed version of the charging current of a single battery with synergetic controller.

are used to filter the sampled current and voltage signals. A zoomed version of the charging current is plotted in Fig. 13. It is seen that the response time is around $0.1 \mathrm{~s}$, which is good enough for this application.

Experiment data and simulation results of the charging currents and battery voltages as three batteries are simultaneously charged are plotted in Fig. 14. Here, the pulse duration of the reference current for each battery is identical (i.e., 33.3\%), and equal to 20 s. It is shown in Fig. 14(a) that three pulse currents are alternately applied to the batteries. Experiment data of the charging currents match the simulation results very well. However, there are some small discrepancies in the battery voltage [See Fig. 14(b)]. This is because the model parameters and initial conditions of the batteries are not exactly identical to the actual ones. Again, the unclean waveforms result from the difference between switching frequency and sampling frequency.

Fig. 15 shows a zoomed version of these charging currents with three control methods: PI control, basic synergetic control, improved synergetic control. It is shown that with PI control the out-of-phase (in practice, not exactly) step changes in the currents of two batteries cause a large variation (approximately $40 \%$ ) in the current of the third battery because the nonsimultaneous step changes of the charging currents may cause a sudden 


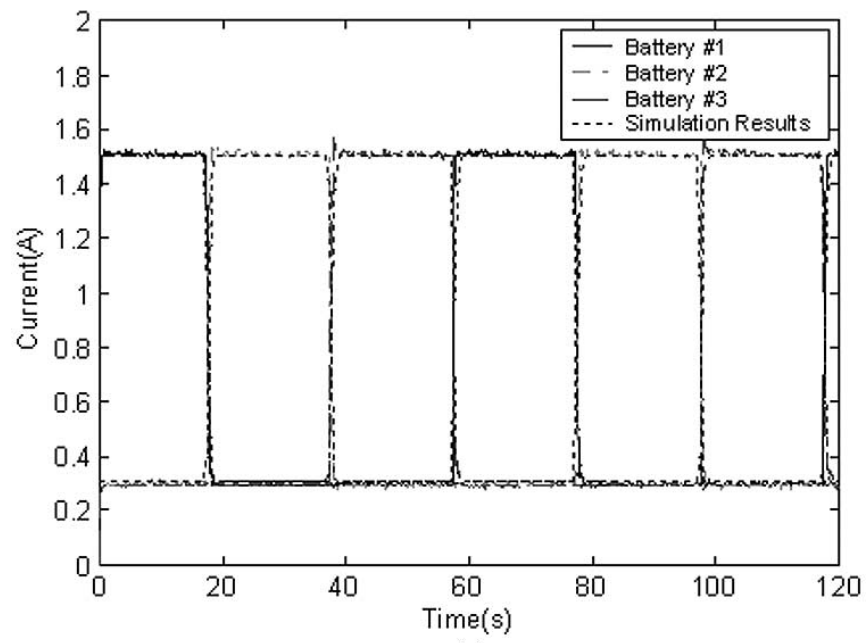

(a)

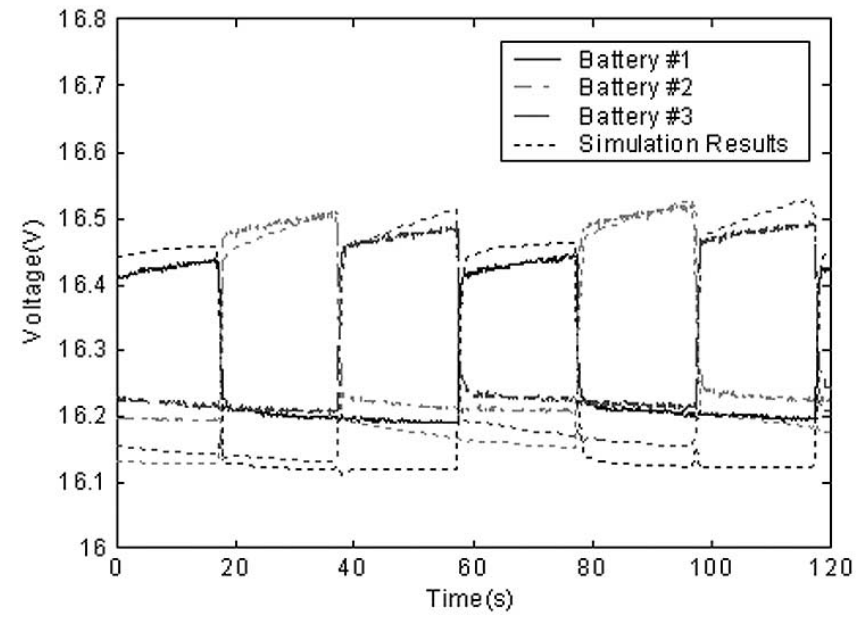

(b)

Fig. 14. Experimental data and simulation results of the charging current and voltages of three batteries with the synergetic controller: (a) current and (b) voltage.

variation in the fuel cell voltage. The response speed of PI control is the lowest among these methods. It is seen that the step changes in the charging currents does not cause significant variation in the current to the other battery with synergetic control laws. Although there is a sudden variation in the voltage of the power source, the synergetic control laws compensate for this variation immediately. There is a steady-state error in the current with basic synergetic control law; however, the integral term in the improved synergetic control law eliminates the steady-state error and increased the response speed to some extent. It is clear that the improved synergetic controller achieves the best transient performances among these methods.

Experiment results validate that the synergetic controller is less sensitive to the parameter variation and input variation and is robust to the pulse output change. The synergetic controller achieves better performances than the linear regulators. Experiment studies also show that the efficiency of the whole system is greater than $90 \%$ and the total charging time for three batteries with pulse current charging protocol is about $25 \%$ shorter than that with de charging protocol.

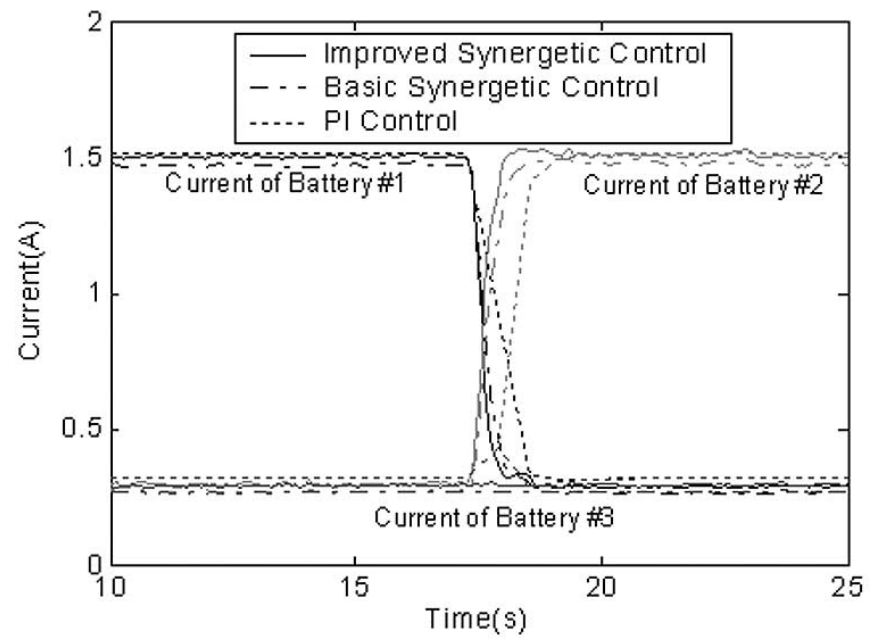

(a)

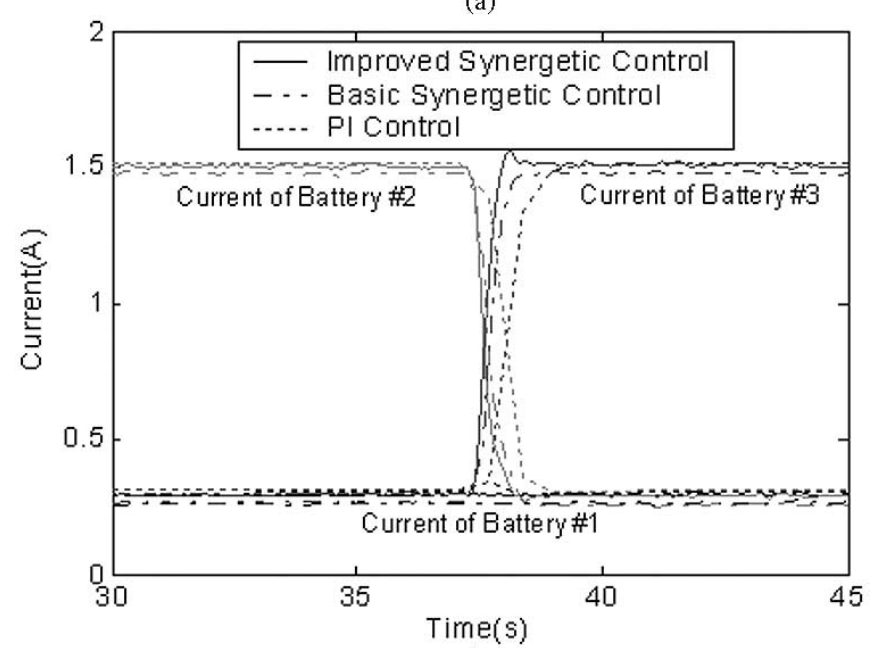

(b)

Fig. 15. Zoomed version of the charging currents of three batteries with synergetic controller.

\section{CONCLUSION}

This paper presents a synergetic controller for pulse current charging of advanced batteries from a fuel cell power source. Pulse-current charging protocol that has been shown to have many advantages over the traditional constant current/constant voltage protocol is applied in a fuel cell powered battery-charging station to reduce the total charging time. Strong nonlinearity and dynamics exist in such system. The synergetic control approach is applied to regulate the buck converters that control the pulse charging currents to the many batteries in this paper. A practical synergetic controller to coordinate the pulse current charging of the batteries is synthesized and discussed. It provides asymptotic stability with respect to the required operating modes, invariance to load variations, and robustness to variation of the input and converter parameters.

The synergetic controller is implemented in Simulink. The dynamic characteristics of the synergetic controller are studied by conducting system simulation and experimental tests. Simulation and experiment results show the synergetic control law is insensitive to the input and parameter variations and the synergetic controller is robust for such nonlinear dynamic system. 
The synergetic controller achieves better performances than the linear regulators.

\section{REFERENCES}

[1] R. J. Brodd, "Overview: Rechargeable battery systems," in Proc. WESCON'93 Conf., 1993, pp. 206-209.

[2] Z. Jiang and R. Dougal, "Control design and testing of a novel fuelcell-powered battery-charging station," in Proc. IEEE Applied Power Electronic Conf., Miami, FL, Feb. 10-14, 2003, pp. 1127-1133.

[3] — "Design and testing of a fuel-cell-powered battery-charging station," J. Power Sources, vol. 115, no. 2, pp. 148-153, Apr. 2003.

[4] —, "Strategy for active power sharing in a fuel-cell-powered charging station for advanced technology batteries," in Proc. 34th IEEE Power Electronics Specialist Conf., Acapulco, Mexico, June 15-19, 2003.

[5] J. Li, E. Murphy, J. Winnick, and P. A. Kohl, "The effects of pulse charging on cycling characteristics of commercial lithium-ion batteries," J. Power Sources, vol. 102, no. 1-2, pp. 302-309, Dec. 2001.

[6] J. Alvarez-Ramirez, I. Cervantes, G. Espinosa-Perez, P. Maya, and A. Morales, "A stable design of PI control for DC-DC converters with an RHS zero," IEEE Trans. Circuits Syst. I, vol. 48, pp. 103-106, Jan. 2001.

[7] B. Choi, B. Cho, F. Lee, and R. Ridley, "Three-Loop control for multimodule converter systems," IEEE Trans. Power Electron., vol. 8, pp. 466-474, Oct. 1993.

[8] R. W. Ashton, J. G. Ciezki, and M. G. Badorf, "The synthesis and hardware validation of DC-to-DC converter feedback controls," in Proc. 29th Annual IEEE Power Electronics Specialists Conf., vol. 1, 1998, pp. 65-71.

[9] V. I. Utkin, "Variable structure system with sliding modes," IEEE Trans. Ind. Electron., vol. AC22, pp. 212-222, Apr. 1977.

[10] K. M. Smedley and S. Cuk, "One-cycle control of switching converters," IEEE Trans. Power Electron., vol. 10, pp. 888-896, Nov. 1995.

[11] A. Kolesnikov et al., Modern Applied Control Theory: Synergetic Approach in Control Theory (in Russia). Moscow: TSURE Press, 2000, vol. 2.

[12] A. Kolesnikov, G. Veselov, A. Monti, F. Ponci, E. Santi, and R. Dougal, "Synergetic synthesis of DC-DC boost converter controllers: Theory and experimental analysis," in Proc. 17th Annual IEEE Applied Power Electronics Conf., vol. 1, Dallas, TX, Apr. 2002, pp. 409-415.

[13] E. Santi, A. Monti, D. Li, K. Proddutur, and R. Dougal, "Synergetic control for DC-DC boost converter: Implementation options," in Proc. 37th IEEE Industry Applications Society Annual Meeting, vol. 2, Oct. 2002, pp. 13-18.
[14] D. Li, K. Proddutur, E. Santi, and A. Monti, "Synergetic control of a boost converter: Theory and experimental verification," in Proc. IEEE Southeast Conf., Apr. 2002, pp. 197-200.

[15] T. Lovett, A. Monti, E. Santi, and R. Dougal, "A multilanguage environment for interactive simulation and development of controls for power electronics," in Proc. IEEE 32nd Annual Power Electronics Specialists Conf., vol. 3, 2001, pp. 1725-1729.

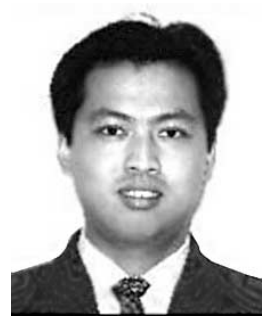

Zhenhua Jiang (S'01-M'03) received the B.Sc. and M.Sc. degrees in electrical engineering from Huazhong University of Science and Technology, Wuhan, China, in 1997 and 2000, respectively, and the $\mathrm{Ph} . \mathrm{D}$. degree in electrical engineering from the University of South Carolina, Columbia, in 2003.

He is currently a Postdoctoral Fellow at the University of South Carolina. His primary research interests include controls, application of power electronics in new energy systems such as electromechanical, photovoltaic and electrochemical energy conversion systems, battery energy storage systems and hybrid fuel cell power sources, and modeling and simulation of interdisciplinary systems.

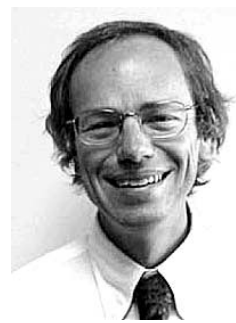

Roger A. Dougal (S'74-M'79-SM'94) received the $\mathrm{Ph} . \mathrm{D}$. degree in electrical engineering from Texas Tech University, Lubbock, in 1983.

He joined the faculty at the University of South Carolina, Columbia, in 1983. He is Director of the Virtual Test Bed Project (VTB) (a multidisciplinary, multiuniversity effort to develop a comprehensive simulation and virtual prototyping environment for advanced power sources and systems, integrating power electronics, electromechanics, electrochemistry, and controls into a common testbed). The VTB is unique in allowing the simulation of multidisciplinary systems by importing models from discipline specific source languages to a common workspace. In addition to modeling and simulation, his expertise includes power electronics, physical electronics, and electrochemical power sources.

Dr. Dougal received the Samuel Litman Distinguished Professor of Engineering Award. 